\title{
Use of extended field-of-view ultrasound imaging in giant primary breast angiosarcoma: a case description
}

\author{
Xinyao Liu^, Shuai Zheng^, Yi Li^, Wen $\mathrm{He}^{\wedge}$ \\ Department of Ultrasound, Beijing Tiantan Hospital, Capital Medical University, Beijing, China \\ Correspondence to: Wen He. Department of Ultrasound, Beijing Tiantan Hospital, Capital Medical University, No. 119, South Fourth Ring Road \\ West, Fengtai District, Beijing 100070, China. Email: ttyyus@sina.com.
}

Submitted Mar 12, 2021. Accepted for publication Aug 03, 2021.

doi: 10.21037/qims-21-280

View this article at: https://dx.doi.org/10.21037/qims-21-280

\section{Introduction}

Primary breast angiosarcoma is a rare malignancy accounting for approximately $0.04 \%$ of malignant breast tumors (1). It is characterized by high malignancy and shows extensive infiltrating growth and hematogenous metastasis $(1,2)$. Breast angiosarcoma is associated with a poor prognosis, and therefore early diagnosis of breast angiosarcoma is critical to reduce the risk of mortality and improve patient outcome (3). Initial misdiagnosis is common in primary breast angiosarcoma, and nonspecific clinical manifestations and imaging features mainly contribute to the misdiagnosis (4).

Here we present a case of a 34-year-old female patient with giant primary breast angiosarcoma. Routine ultrasonography showed diffuse, mixed hyper- and hypoechoic regions without any discrete mass. However, extended field-of-view (EFOV) ultrasound imaging suggested a possible diagnosis of malignancy.

\section{Case presentation}

A 34-year-old woman without a history of breast surgery or irradiation presented with progressive enlargement of the right breast for 4 months. The right breast showed no redness or nipple discharge and was accompanied by pain. On palpation, a large mass involving the right breast was observed, measuring approximately $120 \mathrm{~mm} \times 100 \mathrm{~mm}$, with stiffness, poor mobility and mild tenderness, but without nipple retraction and adhesion to the skin or the chest wall. The left breast was normal on palpation.

Mammography revealed obvious swelling, blurred glands and no apparent abnormal calcification of the right breast. Routine ultrasonography (Samsung Medical, Seoul, Korea) with a 3-12-MHz linear array transducer showed thickened glands with heterogeneous echoes but without any discrete mass (Figure 1A). Color Doppler imaging (Samsung Medical) with a $3-12-\mathrm{MHz}$ linear array transducer revealed hypervascular character (Figure 1B). Spectral Doppler imaging (Samsung Medical) with a $3-12-\mathrm{MHz}$ linear array transducer showed an arterial flow spectrum with a peak systolic velocity (PSV) of $16.78 \mathrm{~cm} / \mathrm{s}$ and resistive index (RI) values of 0.61 (Figure 1C). There was no bilateral axillary lymphadenopathy. However, EFOV ultrasound imaging (General Electric Healthcare, Wauwatosa, WI, USA) with a $5-9-\mathrm{MHz}$ linear array transducer revealed a mixed hyper- and hypoechoic mass that occupied almost the entire right breast. The mass showed angular margins and infiltrated the surrounding structures, which indicated a malignant lesion (Figure 1D). Thus, an ultrasound-guided biopsy was performed. Histopathology suggested that the lesion was a vasogenic tumor with active proliferation and atypia. Combined with the expression of $\mathrm{Ki}-67$ in immunohistochemistry, an aggressive vascular tumor with the possibility of angiosarcoma was considered.

Magnetic resonance imaging (MRI) (Philips Medical

^ ORCID: Xinyao Liu, 0000-0001-5261-5830; Shuai Zheng, 0000-0002-5565-8454; Yi Li, 0000-0001-9087-4833; Wen He, 0000-0002$7177-4498$. 


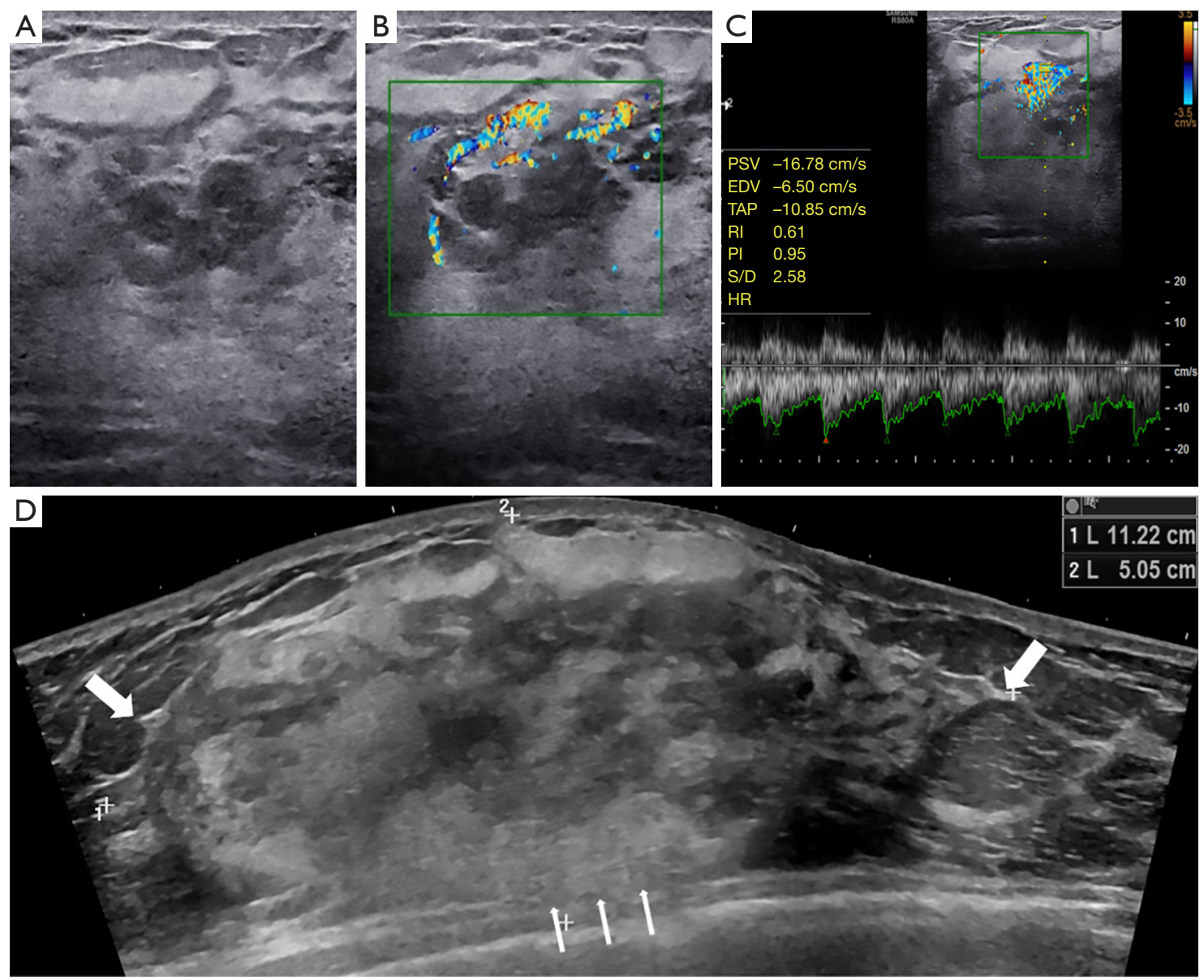

Figure 1 Ultrasonography of the giant breast angiosarcoma. (A) Routine ultrasonography shows a diffuse, mixed hyper- and hypoechoic region. (B) Color Doppler imaging reveals the hypervascular character. (C) Spectral Doppler imaging shows an arterial flow spectrum with PSV of $16.78 \mathrm{~cm} / \mathrm{s}$ and RI values of 0.61. (D) EFOV ultrasound imaging shows a giant, hyper- and hypoechoic mass measuring approximately $112 \mathrm{~mm} \times 50 \mathrm{~mm}$ that occupied almost the entire right breast. The mass shows angular margins (thick arrows) and invasion into adjacent tissues (thin arrows). EFOV, extended field-of-view; PSV, peak systolic velocity; EDV, end-diastolic velocity; TAP, timeaveraged peak velocity; RI, resistance index; PI, pulsatility index; S/D, systolic/diastolic ratio; HR, heart rate.

Systems, Best, Netherlands) in preoperative evaluation revealed a large irregular mass measuring approximately $101 \times 61 \times 57 \mathrm{~mm}$, showing low signal intensity on axial T1weighted $(\mathrm{T} 1 \mathrm{~W})$ image [repetition time $(\mathrm{TR})=571.96 \mathrm{~ms}$; echo time $(\mathrm{TE})=8.04 \mathrm{~ms}$ ] and high signal intensity on axial $\mathrm{T} 2$-weighted (T2W) image ( $\mathrm{TR}=4,428.30 \mathrm{~ms} ; \mathrm{TE}=70.00 \mathrm{~ms}$ ) (Figure $2 A, 2 B$ ). Sagittal dynamic contrast-enhanced MRI $(\mathrm{TR}=5.51 \mathrm{~ms} ; \mathrm{TE}=2.80 \mathrm{~ms})$ in early phase showed a heterogeneously enhanced and diffusely distributed mass (Figure 2C). Time-intensity curves showed rapid initial enhancement and washout on delayed images. The mass appeared hyperintense on axial diffusion-weighted imaging $(\mathrm{DWI})(\mathrm{TR}=9,000.00 \mathrm{~ms} ; \mathrm{TE}=52.35 \mathrm{~ms} ; \mathrm{B}$ value $=800)$
(Figure 2D). MRI demonstrated that the right mammary vessel was thicker than the contralateral vessel.

The patient underwent total mastectomy of the right breast. Histopathology from the mastectomy specimen indicated an intermediate-grade angiosarcoma measuring $190 \mathrm{~mm} \times 90 \mathrm{~mm} \times 60 \mathrm{~mm}$. The tumor infiltrated the surrounding breast tissue with involvement of the subcutis. Immunohistochemistry revealed strong expressions of CD31 and CD34 in the tumor (Figure $3 A$ ). Expression of $\mathrm{Ki}-67$ was up to $40 \%$ in the cellular areas (Figure $3 B$ ).

All procedures performed in studies involving human participants were in accordance with the ethical standards of the institutional and/or national research committee(s) 

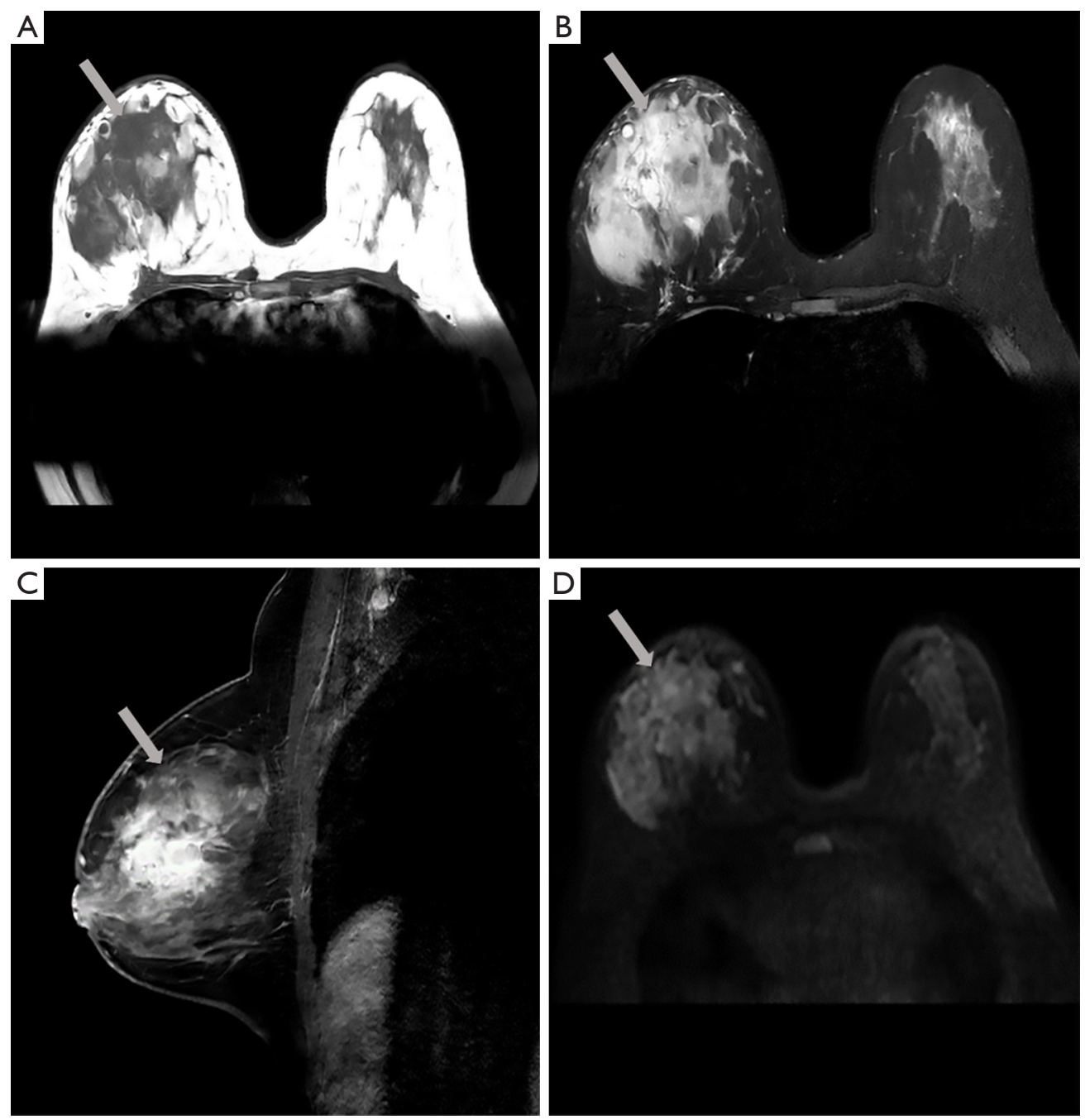

Figure 2 MRI of the giant breast angiosarcoma (arrow). (A) Axial T1W image shows a large, irregular hypointense mass in the right breast ( $\mathrm{TR}=571.96 \mathrm{~ms}$; TE =8.04 ms). (B) Axial T2W MRI with fat saturation shows a heterogeneous hyperintense mass in the right breast (TR =4,428.30 ms; TE =70.00 ms). (C) Sagittal dynamic contrast-enhanced MRI in early phase shows a heterogeneously enhanced and diffusely distributed mass in the right breast ( $\mathrm{TR}=5.51 \mathrm{~ms}$; $\mathrm{TE}=2.80 \mathrm{~ms}$ ). (D) Axial DWI shows a hyperintensity mass in the right breast (TR =9,000.00 ms; TE =52.35 ms; B value =800). MRI, magnetic resonance imaging; T1W, T1-weighted; TR, repetition time; TE, echo time; T2W, T2-weighted; DWI, diffusion-weighted imaging.

and with the Helsinki Declaration (as revised in 2013). Written informed consent was obtained from the patient for publication of this case report and accompanying images. A copy of the written consent is available for review by the editorial office of this journal.

\section{Discussion}

Primary breast angiosarcoma is a rare malignancy that occurs mainly in young women (30 to 40 years) (5).
Angiosarcoma tends to grow rapidly and shows a poor prognosis (6). The median overall survival of primary breast angiosarcoma is 93 months, and the 5 -year survival rate is $44.5 \%$ (2). The mean tumor diameter is around $6 \mathrm{~cm}(5)$. Approximately $17-35 \%$ of patients present with blue skin discoloration, which is a specific clinical manifestation (7). Blood metastasis rather than lymphatic metastasis is more common in mammary angiosarcoma (1). Lung, liver, bones and skin are common sites of metastasis of breast angiosarcoma (1). 


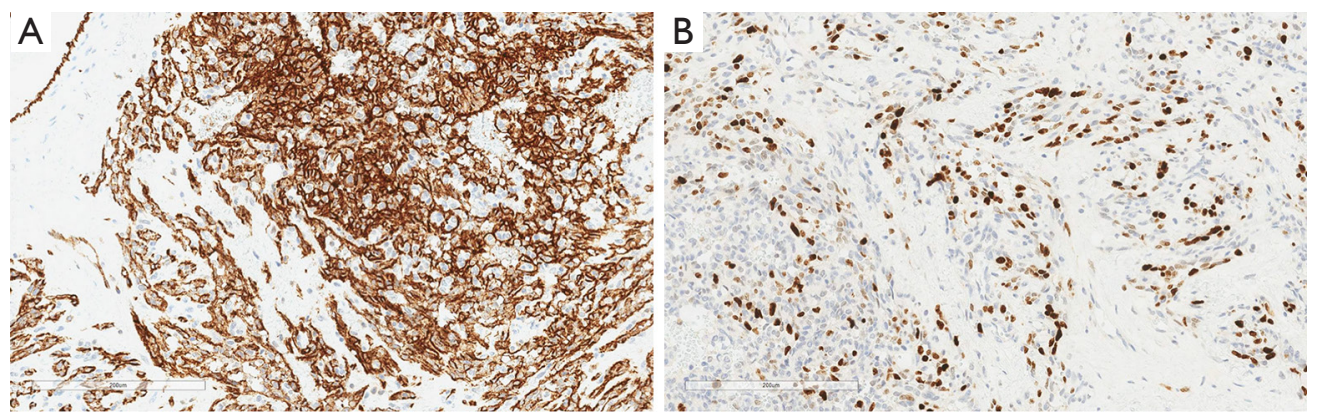

Figure 3 Immunohistochemistry of the giant breast angiosarcoma. (A) Immunohistochemical staining reveals strong expression of CD31 (magnification, $\times 20$ ). Scale bar, $200 \mu \mathrm{m}$. (B) Immunohistochemical staining shows that expression of Ki-67 is up to $40 \%$ in the cellular areas (magnification, $\times 20$ ). Scale bar, $200 \mu \mathrm{m}$.

On the basis of histopathology, angiosarcomas are subclassified into three grades (8). Grade I is a well differentiated tumor and contains open anastomosing vascular channels lined by endothelial cells that dissect through stroma. Grade II is the intermediate grade tumor and additionally shows papillary formations. Grade III is a poorly differentiated tumor that shows prominently solid and spindle cell areas and additionally presents hemorrhage and necrosis.

Breast angiosarcoma might be invisible on mammography because of the dense breast tissue in young women. The mammographic findings for angiosarcoma that are visible are often nonspecific. Visible lesions may present as round to oval or irregular in shape and may show circumscribed or obscure boundaries (1). Some cases may manifest with focal asymmetry, which reflects the subtle infiltrating nature of angiosarcoma (9).

Primary breast angiosarcoma may appear as a hypoechoic or hyperechoic mass with well-circumscribed or indistinct margins and without posterior acoustic phenomena on ultrasonography $(1,9)$. Liquid anechoic regions associated with hemorrhagic and necrotic changes are also observed (10). Color Doppler imaging reveals the hypervascular character of breast angiosarcoma (9). Approximately 54\% of mammary angiosarcoma are hyperechoic or mixed hyperand hypoechoic (9). This appearance may reflect the vascular nature of breast angiosarcoma and the multiple interfaces of the vascular channels (9). In cases that show this appearance, a possible breast angiosarcoma diagnosis may be suggested (9).

As observed in our current case, breast angiosarcoma can also appear as diffuse, mixed hyper- and hypoechoic regions without any discrete mass at routine ultrasonography (9). We first considered that the sonographic features might be related to inflammatory changes. Given that the abnormal region was large on palpation and routine ultrasonography was not able to show the entire lesion, EFOV ultrasound imaging was used for further workup. The full lesion image presented by EFOV ultrasound imaging alerted us to the possible diagnosis of malignancy. Using a small-foot-print transducer when imaging a giant mass may result in misdiagnosis and missed diagnosis if the diffuse heterogeneous echoes without any discrete mass are neglected by a sonographer searching for a mass. Sonographers should be aware of the possibility that the tumor is too large to be fully shown in the image when they encounter diffuse regions without a discrete mass at routine ultrasonography.

Previous studies showed that the abnormal heterogeneous echoes without any discrete mass are generally related to large tumors (9). This appearance may reflect the subtle infiltrating nature of breast angiosarcomas, which tend to ramify within the breast tissue (9). This feature is especially significant when imaging giant angiosarcoma. Evaluating large breast masses by routine ultrasonography is difficult because these large breast masses cannot be completely defined or measured when all margins are not included in the field of view. EFOV ultrasound imaging can build single frame 2D mode sonographic images into larger composite images using real-time scanning, providing the sonographer with a larger field of view and a new perspective for evaluating lesions (11). This imaging approach can depict large masses from a broad perspective, clearly showing the anatomical relationship between the lesion and surrounding tissues and allowing a precise overview and measurement (12). The EFOV technique provides a complete evaluation of lesion size and mass margin (11). In $21 \%$ of masses 
evaluated, the conspicuity of subtle lesions was improved because the lesion could be contrasted with a larger area of surrounding normal tissue (11). EFOV ultrasound imaging can fully show the extent, morphology and surrounding structures of the giant mass and help physicians make the appropriate clinical decision on further examination and treatment.

Primary breast angiosarcoma presents as a heterogeneous mass with low intensity on T1W image and high intensity on T2W image (13). Enhancement of the mass on MRI is associated with tumor grade. Progressive enhancement is observed in low-grade angiosarcomas, while rapid enhancement and washout with usual visualization of large draining vessels are observed in high-grade angiosarcomas (13). Higher-grade angiosarcomas might show irregular areas of high T1 signal, which reflect venous lakes or hemorrhagic zones (13). MRI is a useful imaging test for characterizing mammary angiosarcomas, as it can determine the lesion extent and visualize the vascular nature of tumors, helping in diagnosis and planning surgery.

The differential diagnosis of primary breast angiosarcoma mainly includes mastitis or breast cancer (4). On ultrasonography, mastitis is observed as irregular hypoechoic lesions with or without mobile internal echoes (1). EFOV ultrasound imaging can fully show the extent, morphology and surrounding structures of the lesions, helping in the differential diagnosis. On contrast-enhanced MRI, most nonpuerperal mastitis manifest as non-mass-like lesions with heterogeneous signal intensity (14). The signs of rim or rim-like enhancement on contrast-enhanced MRI, with central hypointensity areas showing as hyperintensity on $\mathrm{T} 2 \mathrm{~W}$ images, are suggestive of the possibility of nonpuerperal mastitis (14). MRI can differentiate primary breast angiosarcoma from mastitis when T1W image shows high signal intensity due to hemorrhage or venous lakes (15). Breast angiosarcoma does not appear as spiculated contours or microcalcifications, which are both typical of breast cancer on mammography (16). In addition, breast cancer shows posterior acoustic phenomena on ultrasonography and axillary lymph nodal involvement is common, which are rare in primary breast angiosarcoma (1). Angiosarcomas may show irregular areas of high T1 signal from hemorrhage or venous lakes, which is different from breast cancer (13).

The general treatment of primary breast angiosarcoma is total mastectomy (5), and adjuvant therapy may improve patient survival (1). The tumor size, histologic characteristics, and surgical margin are associated with prognosis of patients with primary breast angiosarcoma (17).
Well-differentiated angiosarcoma that is less than $5 \mathrm{~cm}$ in size, with a lower grade and a negative margin, tends to have a better prognosis (17).

In conclusion, primary breast angiosarcoma is a rapidgrowing malignancy that is mostly observed in young women. Mammography and ultrasonography are the firstline imaging modalities. However, dense breast tissue may often lead to the tumor being missed on mammography. EFOV ultrasound imaging is helpful in diagnosing primary breast angiosarcoma. Sonographers should use EFOV ultrasound imaging to further examine the breast when they encounter diffuse lesions without a discrete mass at routine ultrasonography. EFOV ultrasound imaging can fully show the extent, morphology and surrounding structures of the giant mass, indicating a probable diagnosis of the malignant tumor and helping in the diagnosis and treatment plan. Therefore, EFOV ultrasound imaging may reduce misdiagnosis and missed diagnosis of primary breast angiosarcoma and improve the accuracy and convenience of diagnosis to some extent. MRI may narrow the differential diagnosis, indicate the possibility of angiosarcoma, suggest tumor grade and help establish a surgery plan. The final diagnosis depends on surgical resection for histopathological examination.

\section{Acknowledgments}

We thank Gabrielle White Wolf, PhD, from Liwen Bianji (Edanz) (www.liwenbianji.cn/), for editing the English text of a draft of this manuscript.

Funding: This work was supported by the National Natural Science Foundation of China (ID 8173000716).

\section{Footnote}

Conflicts of Interest: All authors have completed the ICMJE uniform disclosure form (available at https://dx.doi. org/10.21037/qims-21-280). The authors have no conflicts of interest to declare.

Ethical Statement: The authors are accountable for all aspects of the work in ensuring that questions related to the accuracy or integrity of any part of the work are appropriately investigated and resolved. All procedures performed in studies involving human participants were in accordance with the ethical standards of the institutional and/or national research committee(s) and with the Helsinki Declaration (as revised in 2013). Written informed consent 
was obtained from the patient for publication of this case report and accompanying images. A copy of the written consent is available for review by the editorial office of this journal.

Open Access Statement: This is an Open Access article distributed in accordance with the Creative Commons Attribution-NonCommercial-NoDerivs 4.0 International License (CC BY-NC-ND 4.0), which permits the noncommercial replication and distribution of the article with the strict proviso that no changes or edits are made and the original work is properly cited (including links to both the formal publication through the relevant DOI and the license). See: https://creativecommons.org/licenses/by-nc-nd/4.0/.

\section{References}

1. Varghese B, Deshpande P, Dixit S, Koppiker CB, Jalnapurkar N. Primary angiosarcoma of the breast: a case report. J Radiol Case Rep 2019;13:15-25.

2. Yin M, Wang W, Drabick JJ, Harold HA. Prognosis and treatment of non-metastatic primary and secondary breast angiosarcoma: a comparative study. BMC Cancer 2017;17:295.

3. Mendoza R, Loukeris K. Primary epithelioid angiosarcoma of the breast: a rare and challenging biopsy diagnosis. Am J Case Rep 2019;20:437-40.

4. Mumin NA, Rahmat K, Hamid MTR, Ng WL, Chan WY, Cheah XY, See MH, Yip CH. Primary breast angiosarcoma: utilisation of pre-surgical magnetic resonance imaging (MRI) for accurate tumour characterization and planning - a case report and literature review. Curr Med Imaging 2021;17:552-8.

5. Abdelhady AM, Neamaalla S, Gittens AS, Germaine P. Primary angiosarcoma of the breast: Case report of a rare vascular tumor. Radiol Case Rep 2020;15:339-43.

6. Arora TK, Terracina KP, Soong J, Idowu MO, Takabe K. Primary and secondary angiosarcoma of the breast. Gland Surg 2014;3:28-34.

7. Marchant LK, Orel SG, Perez-Jaffe LA, Reynolds C,

Cite this article as: Liu X, Zheng S, Li Y, He W. Use of extended field-of-view ultrasound imaging in giant primary breast angiosarcoma: a case description. Quant Imaging Med Surg 2022;12(1):868-873. doi: 10.21037/qims-21-280
Schnall MD. Bilateral angiosarcoma of the breast on MR imaging. AJR Am J Roentgenol 1997;169:1009-10.

8. Donnell RM, Rosen PP, Lieberman PH, Kaufman RJ, Kay S, Braun DW Jr, Kinne DW. Angiosarcoma and other vascular tumors of the breast. Am J Surg Pathol 1981;5:629-42.

9. Yang WT, Hennessy BT, Dryden MJ, Valero V, Hunt KK, Krishnamurthy S. Mammary angiosarcomas: imaging findings in 24 patients. Radiology 2007;242:725-34.

10. Mouhoub M, Miry A, Haloui A, Karich N, Kamaoui I, Benkirane S, Bennani A. Primary angiosarcoma of the breast: a case report. Pan Afr Med J 2019;33:134.

11. Ghate SV, Soo MS, Mengoni PM. Extended field-ofview two-dimensional ultrasonography of the breast: improvement in lesion documentation. J Ultrasound Med 1999;18:597-601.

12. Szebeni A, Rahóty P, Besznyák I. Clinical validity of new ultrasound methods in the differential diagnosis of breast diseases. Breast 2002;11:489-95.

13. O'Neill AC, D'Arcy C, McDermott E, O'Doherty A, Quinn C, McNally S. Magnetic resonance imaging appearances in primary and secondary angiosarcoma of the breast. J Med Imaging Radiat Oncol 2014;58:208-12.

14. Liu H, Peng W. Morphological manifestations of nonpuerperal mastitis on magnetic resonance imaging. J Magn Reson Imaging 2011;33:1369-74.

15. Yang OO, Lan T, He JL, Xu HB, Hao L, Shu C, Hu ZJ, Luo H. Magnetic resonance imaging and contrastenhanced ultrasound findings of a recurrent primary breast angiosarcoma: a case report. Medicine (Baltimore) 2021;100:e24625.

16. Zincone GE, Perego P, Rossi GM, Bovo G. A case of breast angiosarcoma: diagnostic imaging and review of the literature. Tumori 1995;81:387-96.

17. Sasahara A, Tanabe M, Hayashi K, Konishi T, Oya M, Sakiyama K, Morizono A, Harada M, Otsuji K, Ishibashi Y, Sato A, Kikuchi Y, Niwa T, Hinata M, Nishioka K, Seto Y. A case of primary breast angiosarcoma with multiple discontinuous small lesions. Surg Case Rep 2019;5:157. 